\title{
Applications and advances of metabolite biosensors for metabolic engineering
}

Authors: Di Liu ${ }^{\mathrm{a}}$, Trent Evans ${ }^{\mathrm{b}}$, Fuzhong Zhang ${ }^{\mathrm{a}, \mathrm{b}^{\star}}$

${ }^{a}$ Department of Energy, Environmental and Chemical Engineering,

${ }^{b}$ Division of Biological \& Biomedical Sciences,

Washington University in St. Louis, Saint Louis, MO 63130, USA

\section{"Correspondence:}

Dr. Fuzhong Zhang

Department of Energy, Environmental \& Chemical Engineering, Washington

University

1 Brookings Drive, St. Louis, MO, 63130, USA

fzhang@seas.wustl.edu

Tel: 314-935-7671 


\section{Abstract}

Quantification and regulation of pathway metabolites is crucial for optimization of microbial production bioprocesses. Genetically encoded biosensors provide the means to couple metabolite sensing to several outputs invaluable for metabolic engineering. These include semi-quantification of metabolite concentrations to screen or select strains with desirable metabolite characteristics, and construction of dynamic metabolite-regulated pathways to enhance production. Taking inspiration from naturally occurring systems, biosensor functions are based on highly diverse mechanisms including metabolite responsive transcription factors, two component systems, cellular stress responses, regulatory RNAs, and protein activities. We review recent developments in biosensors in each of these mechanistic classes, with considerations towards how these sensors are engineered, how new sensing mechanisms have led to improved function, and the advantages and disadvantages of each of these sensing mechanisms in relevant applications. We particularly highlight recent examples directly using biosensors to improve microbial production, and the great potential for biosensors to further inform metabolic engineering practices.

\section{Key words}

Metabolite biosensor, synthetic biology, metabolic engineering, pathway optimization, dynamic pathway regulation 


\section{Abbreviations}

MRTF, metabolite-responsive transcription factor

MAGE, multiplex automated genome engineering

FAEE, fatty acid ethyl ester

ACC, acetyl-CoA carboxylase

TCS, two-component system

HK, histidine kinase

$R R$, response regulator

FPP, farnesyl pyrophosphate

FRET, forster resonance energy transfer

FPX, fluorescent protein exchange

PBP, periplasmic binding protein

IPP, isopentenyl diphosphate

MCFA, medium-chain fatty acid 


\section{Introduction}

Advances in metabolic engineering have enabled microbial production of a wide variety of valuable compounds, providing alternative synthesis routes for chemicals including biofuels, pharmaceuticals, nutraceuticals, bulk chemicals, and materials. To produce these valuable compounds, efficient biosynthetic pathways must be constructed in appropriate hosts, which often requires extensive optimization to reach economically viable titers, yields, and productivities. The cycle of repeatedly tuning pathway parameters and evaluating production is laborious and time-consuming. Synthetic biology is a fast-growing field that develops new tools for biological engineering, fulfilling the need for efficient pathway optimization. It has proven effective in increasing process predictability and throughput as well as in creating new strategies to optimize biosynthetic pathways. Among these new tools, biosensors represent a significant contribution from synthetic biology and have been increasingly used in metabolic engineering. Here, we review recent work on the development of metabolite biosensors and their applications for metabolic engineering.

Biosensors are ubiquitous in nature and have evolved to detect both environmental signals (e.g. temperature, $\mathrm{pH}$, oxygen) and intra- and extracellular metabolites. These sensed signals are coupled with actuator outputs to modify the transcription, translation, and protein activities of cells. A crucial consideration in synthetic biology and metabolic engineering is that these natural biosensing machineries have evolved to maximize evolutionary 
fitness rather than overproduction of any metabolite. However, detailed knowledge of the sensing mechanisms and endogenous functions of these biosensors serve as a valuable starting point to co-opt them for overproduction goals.

This review will focus on "metabolite biosensors" developed for metabolic engineering applications. We define "metabolite biosensors" as geneticallyencoded protein or RNA-based sensors that interact with a metabolite to generate an actuator output. The output domain of a metabolite biosensor generates detectable phenotypes through modulating transcription rates, translation rates, or post-translational parameters to control protein expression or activity. Over the past few decades, metabolite biosensors have drawn tremendous attention and have several applications in metabolic engineering (Figure 1). First, biosensors can be coupled to readable outputs such as fluorescence to semi-quantitatively report the concentration of a target compound. This approach is frequently used for high-throughput screening of high-producing strains and features distinct advantages over conventional methods such as gas chromatography (GC) and highperformance liquid chromatography (HPLC): 1) Biosensor-mediated quantification avoids time-consuming sample preparation and has much higher throughput than conventional chromatographic techniques; 2) metabolite biosensors are more suitable for detecting labile and low abundant metabolites such as acyl-phosphate, acyl-diphosphate, aldehyde, and acylCoAs, which are difficult to measure accurately by conventional methods; 3) metabolite biosensors allow real-time monitoring of metabolite dynamics in 
living cells, which is impossible to study using chromatographic methods. These reporter outputs may also help coordinate complementary manipulations of the culture environment itself (mixing, nutrient addition, timing of harvest) to further improve production (Polizzi and Kontoravdi, 2015). Second, biosensors can be engineered to couple the sensing of a desirable product or intermediate metabolite with a fitness advantage for the cell by expressing a gene necessary for survival under selective conditions (Dietrich et al., 2013; Raman et al., 2014). The difference in cell growth allows direct enrichment of fast-growing cells from mutant libraries, which allows an easy selection for desirable production characteristics. Third, metabolite biosensors can also be used to control metabolic flux dynamically (Dahl et al., 2013; Liu et al., 2013; Zhang et al., 2012; Zhou and Zeng, 2015). The actuator can be designed to tune pathway enzyme expression or post-translational parameters in response to the level of the relevant metabolite, allowing for dynamic control of pathway activity based on the cellular metabolic state. As a result, the pathway is dynamically balanced, which not only reduces toxic intermediate accumulation but also saves carbon and energy that is otherwise diverted to synthesize unnecessary proteins or intermediates. Overall, the emerging tools to engineer biosensors and their applications towards metabolic engineering have greatly advanced microbial production of a variety of chemicals.

While biosensors have been reviewed previously (Gredell et al., 2012; Michener et al., 2012; Palmer et al., 2011; Schallmey et al., 2014; Su et al., 2011; Zhang and Keasling, 2011), this review focuses on recent advances in 
metabolite biosensors and emphasizes their applications for metabolic engineering. Here we classify metabolite biosensors into five categories based on their diverse mechanisms of sensing and functional output, including 1) metabolite-responsive transcription factors, 2) two-component systems, 3) cellular stress response, 4) regulatory RNAs, and 5) protein activities. Biosensors that detect environmental signals have been previously reviewed (Salis et al., 2009; Van Dorst et al., 2010; Zhang and Keasling, 2011) and will not be discussed here.

\section{Metabolite biosensors}

\subsection{Biosensors Based on Metabolite-Responsive Transcription Factors}

In nature, transcription factors regulate gene expression by specific binding to the chromosomal DNA, blocking or promoting transcription by RNA polymerase. Among these, some transcription factors can be activated or deactivated by small molecules through ligand binding, phosphorylation, or interaction with other regulatory elements. Here we will focus on transcription factors that respond to metabolites.

Metabolite-responsive transcription factors (MRTFs) have evolved to interact with various metabolites. Escherichia coli, for example, has more than 230 transcription factors (Binder et al., 2012), which sense a wide variety of metabolites, including sugars, sugar phosphates, amino acids, and lipids. Natural MRTFs have been extensively explored to engineer biosensors for 
metabolic engineering applications. Typically, metabolite-responsive promoters with tunable output dynamic ranges can be engineered by inserting the cognate operator of a MRTF into a synthetic promoter to regulate genes of interest (Figure 2a, 2b). Using this strategy, biosensors that respond to a variety of metabolites have been created, including sensors for butanol (Dietrich et al., 2013), alkanes (Reed et al., 2012), malonyl-CoA (Liu et al., 2013; Xu et al., 2014), acyl-CoA (Zhang et al., 2012) and aromatic aldehyde (Fiorentino et al., 2009). The primary use of MRTF sensors is to screen for high-producing strains from a library of natural or engineered strains, as demonstrated in the production of several chemicals, including mevalonate (Tang and Cirino, 2011), L-lysine (Binder et al., 2012), and triacetic acid lactone (Tang et al., 2013). This approach becomes particularly powerful when coupled with fluorescence-activated cell sorting (FACS). In one example, an eyfp was cloned 3' of a Corynebacterium glutamicum promoter that is regulated by an endogenous transcription factor Lrp, which can detect Lmethionine and several branched-chain amino acids, including L-valine, Lleucine and L-isoleucine (Mustafi et al., 2012). Using chemical mutagens, random mutations were introduced to the Corynebacterium glutamicum strains, which carry the sensor plasmid. Cells were cultivated and screened by FACS, and the ones with enhanced fluorescence were isolated and recultivated to enrich the high-producing strains. Mutants that produce up to a total of $11 \mathrm{mM}$ branched-chain amino acids were identified. In addition, MRTFs have also been used to control genes associated with cell growth/survival for selection (Dietrich et al., 2013; Raman et al., 2014). In a recent paper, Raman et al used MRTF-regulated promoters to control the 
expression of TolC, a protein that allows both positive and negative selections when supplemented with sodium dodecyl-sulfate (SDS) and colicin E1, respectively. While positive selections were needed to select for highproducing strains generated by multiplex automated genome engineering (MAGE), negative selections were used to eliminate the false positives caused by mutations that deactivate the sensor-selection system. By alternating between negative and positive cycles, the authors demonstrated enhanced production for both naringenin and glucaric acid (Raman et al., 2014). Overall, biosensor-mediated high-throughput screening and selection methods drastically shorten the time required to analyze mutant cells, enhancing the power of evolutionary approaches. In addition, MRTF-based biosensors have also been used to dynamically regulate metabolic flux. One of the earliest examples of using the MRTF sensor for dynamic regulation involves a fatty acyl-CoA biosensor FadR (Zhang et al., 2012). FadR naturally regulates several genes in $E$. coli fatty acid biosynthesis and degradation. In a fatty acid ethyl ester (FAEE)-producing strain, the cognate actuator of FadR was engineered to upregulate acyl-CoA biosynthesis, ethanol production, and the expression of a wax ester synthase, which condenses ethanol and acylCoAs to FAEEs (Figure 2c). This allows the downstream pathway to be activated only when there is sufficient acyl-CoA, preventing the production of unnecessary proteins and ethanol at the early stage of fermentation. As a result, the FAEE titer was increased 3-fold, reaching $1.5 \mathrm{~g} / \mathrm{L}$. In addition, other studies constructed negative feedback circuits to dynamically regulate fatty acid biosynthesis using a malonyl-CoA-responsive MRTF (Liu et al., 2013; Xu et al., 2014). Malonyl-CoA is synthesized from acetyl-CoA by acetyl-CoA 
carboxylase (encoded by $a c c$ ). Expression of acc improves fatty acid production, but acc overexpression also inhibits cell growth. To alleviate the toxicity from acc overexpression while maintaining high malonyl-CoA concentrations, malonyl-CoA biosensors were used to dynamically downregulate acc expression when cells accumulate high malonyl-CoA levels. These sensor-enabled dynamic regulations enhanced fatty acid production dramatically, particularly when coupled with upregulation of fatty acid chain elongation genes.

So far, most MRTF-based biosensors have relied on naturally existing transcription factors, and these sensors are usually highly specific to their corresponding metabolites. To allow broader utilization, the specificity of an MRTF needs to be altered to detect a metabolite of interest to which no natural sensor exists. The specificity of a MRTF can be altered using various protein engineering approaches (rational design, evolution, etc.). For example, a biosensor for mevalonate (a precursor of isoprenoid biosynthesis) was developed based on AraC, which naturally recognizes arabinose (Tang and Cirino, 2011). In this work, the specificity of AraC was modified by subjecting five residues of its metabolite-binding pocket to saturation mutagenesis. To select for mevalonate-responsive AraC variants, a gfp gene was placed under the control of a $\mathrm{P}_{\mathrm{BAD}}$ promoter. FACS was then used to isolate clones with higher GFP expression in the presence of mevalonate. Another method to create novel MRTFs is through engineering chimeric proteins. In one example, an isopentenyl diphosphate (IPP) biosensor was created by fusing the AraC DNA binding domain and the linker with IPP isomerase Idi (Chou and 
Keasling, 2013). These general approaches are useful to develop sensors for a variety of compounds, as demonstrated for triacetic acid lactone (Tang et al., 2013), D-arabinose (Tang et al., 2008), fructose, ribose (Meinhardt et al., 2012; Shis et al., 2014), etc.

\subsection{Two-Component System Biosensors}

Two-component systems (TCS) prototypically communicate environmental signals to intracellular, transcriptional changes. A TCS biosensor usually contains a sensor protein with histidine kinase (HK) activity coupled via phosphotransfer (or similar) activity to a cytosolic response regulator (RR), often a transcription factor (Figure 3a). HKs often contain membrane localization domains so that some TCSs can sense extracellular metabolites, which is not possible for many MRTFs if the metabolite is membraneimpermeable. Extracellular metabolite sensing is particularly relevant when products are pumped or secreted after synthesis, or in co-cultures (Shong et al., 2012; Wen et al., 2014; Zhou et al., 2015) where a signaling metabolite produced by one strain cannot cross the membrane of another. One recent demonstration of the utility of TCS in sensing an extracellular metabolite is the creation of a malate-sensing $\mathrm{HK}$ protein bound to an EnvZ response regulator, resulting in screenable GFP output proportional to malate concentration (Ganesh et al., 2015). Importantly, this novel grafting of a malate sensing domain into an endogenous $\mathrm{HK}$ allowed a greatly expanded range of malate sensitivity compared to an endogenous malate-sensing TCS and illustrates how TCSs can be reengineered as biosensors. Additional attractive features 
of TCS-based biosensors include the enormous pre-existing diversity in HK sensor and RR components as well as the HK-RR interchangeability arising from high interface domain homology. However, challenges lie in maintaining efficient and specific phosphotransfer between domains while preventing offtarget HK-RR interaction (Capra and Laub, 2012). Whitaker et al. introduced the concept of increasing the fidelity of phosphotransfer through inserting scaffold-binding domains into each TCS component (Whitaker et al., 2012). Tight coupling of components on the protein scaffold was achieved, leading to improved specificity. In another example, Hsiao et al. created a twocomponent circuit with negative feedback regulation. When an input RFPscaffolding protein was sensed, the $H K$ and $R R$ bound and initiated a phosphotransfer, resulting in the transcription of an antiscaffold-YFP protein (Hsiao et al., 2015), which sequestered the free RFP-scaffolding protein and at the same time expressed a yellow fluorescent protein (YFP). Thus, fluorescence-monitorable negative feedback occurred through RFP neutralization and dissociation of the TCS. Adaptation of such systems to sense desirable metabolites in a production pathway could prove useful in dynamic metabolic regulation.

Beyond two-component systems, multiple components can be engineered to detect extracellular metabolites. A recent study used G-protein coupled receptors (GPCRs) to sense medium-chain fatty acid and coupled it via the yeast mating pathway to a specific transcription factor, which activates the transcription of a GFP, resulting in sensors that detect even-chain C8-C12 fatty acids with dynamic range up to 17-fold in Saccharomyces cerevisiae 
(Mukherjee et al., 2015). Similar sensors can be potentially created by engineering other signaling pathways.

\subsection{Biosensors Based on Stress Response}

Naturally, cells possess delicate mechanisms to modulate gene transcription in response to various stresses. The biosynthesis of heterologous metabolites or the over-accumulation of native metabolites to toxic levels often lead to stress responses, which can be identified using genome-wide transcriptomic tools, such as RNAseq or RNA microarray (Alsaker et al., 2010; Rutherford et al., 2010) (Figure 3b). Based on this mechanism, a series of stress-responsebased sensors for the accumulation of farnesyl pyrophosphate (FPP) were identified. FPP is an important intermediate in the synthesis of terpenes and terpenoids. The promoters of the genes that are down-regulated in the presence of FPP were used to control the biosynthesis of FPP, forming a negative feedback loop, whereas the promoters that are activated by FPP were used to control the expression of an amorphadiene synthase, which converts FPP to amorphadiene (Figure 3c). This dynamic regulation built from stress-response biosensors increased the overproduction of amorphadiene by 2-fold, reaching $1.2 \mathrm{~g} / \mathrm{L}$ (Dahl et al., 2013). This strategy can potentially be used to identify biosensors for any metabolite that causes cellular transcriptomic changes. However, because metabolite-induced stress response can involve multi-layer interactions, it is likely that the identified

promoter also responds to other metabolites or signals. Furthermore, the 
mechanism of stress response varies with metabolites and is difficult to study, making it difficult to fine tune the response curve.

\subsection{Biosensors Based on RNAs}

While MRTF-, TCS-, and stress response-based sensors control transcription initiation, RNA sensors provide regulation of transcription termination, translation initiation, and RNA stability. Riboswitches and aptazymes are major RNA sensors that consist of an aptamer domain to interact with a ligand and an actuator domain to transduce the signal into a change in gene expression.

At the transcriptional level, ligand binding to the aptamer domain can be engineered to either facilitate or disrupt the formation of a terminator, which prevents the synthesis of long mRNAs, creating transcriptional repression or activation, respectively (Figure 4a). This mechanism has been used to engineer biosensors that respond to different metabolites such as folinic acid and theophylline (Trausch et al., 2011; Wachsmuth et al., 2013). To increase output dynamic range (the ratio of a signal in the presence of metabolite to that in the absence of metabolite), tandem copies of the same riboswitches can be combined in a single transcription unit to increase the number of metabolite-controlled terminators (Wachsmuth et al., 2013). Meanwhile, by combining riboswitches with different metabolite specificities in one transcript, Boolean logic gates can be created to control gene expression based on 
multiple metabolites, which is useful to sense multiple cellular input signals (Wachsmuth et al., 2013).

At the translational level, riboswitches sense metabolites and modulate the secondary structure of mRNAs to either present or sequester the RBS sequence from the ribosome (Figure 4b), a strategy predominantly used by prokaryotes to regulate translation initiation (Chang et al., 2012). Based on this mechanism, synthetic riboswitches have been engineered to sense a series of metabolites, including theophylline (Topp et al., 2010), ammeline (Dixon et al., 2010), and thiamine pyrophosphate (Muranaka et al., 2009). In one study, a natural AdoCbl-responsive (also known as coenzyme $B_{12}$ ) riboswitch was modified to create synthetic AdoCbl biosensors, which has been suggested to modulate translation by AdoCbl-induced sequestration of the RBS. This biosensor was then used to study the effects of gene deletions and the expression of heterologous genes to reveal the metabolism and transport of AdoCbl (Fowler et al., 2010). The application of biosensors to assess genetic mutants can potentially be used to identify not only the genes involved in the synthesis, consumption, and transport of the metabolite, but also roles of metabolic regulatory network in response to induced perturbations. The obtained information would provide putative chromosomal genetic targets to enhance the production of a metabolic pathway.

Further, metabolite biosensors can be engineered based on metabolitecontrolled transcript stability through aptazymes, in which an aptamer is linked to a hammerhead ribozyme (Figure 4c). One example is the theophylline- 
responsive aptazymes. Upon cloning this aptazyme to the 3' untranslated region of a fluorescent reporter gene (GFP), the presence of theophylline triggers a conformational change of the aptazyme, leading to decreased mRNA self-cleavage activity and enhanced GFP expression. This aptazyme sensor was then used to screen for a library of caffeine demethylases, a key enzyme in the biosynthesis of theophylline, resulting in a 33-fold enhancement in enzyme activity and a 22-fold enhancement in theophylline production (Michener and Smolke, 2012). Another mechanism involves metabolite-controlled activity of an RNase III enzyme, Rnt1p (Figure 4d), as demonstrated in the case of a theophylline-responsive biosensor (Babiskin and Smolke, 2011). The binding of theophylline to the aptamer triggers a structural change that inhibits Rnt1p processing, thus increasing the stability of the transcript. A nice feature of Rnt1p hairpins is that the binding module and the cleavage module function independently, which provides facile strategies to tune the biosensor response, including tuning 1) the ligandaptamer interaction, 2) binding affinity of Rnt1p to the hairpin, and 3) the Rnt1p cleavage rate.

In addition, riboswitches can be also engineered in eukaryotic cells to control pre-mRNA splicing (Figure 4e). Metabolite induced and inhibited splicing have been identified in nature and used as metabolite sensors in engineered systems. For example, a tetracycline sensor was created by incorporating a tetracycline aptamer in the 5' splice site in such a way that adding tetracycline facilitates the formation of an aptamer-tetracycline complex structure that inhibits splicing (Weigand and Suess, 2007). The 
dynamic range of the sensor was tuned by modulating the position and stability of the aptamer and by combining multiple aptamers in the same transcript. Another mechanism to engineer metabolite biosensors in eukaryotes is through microRNA-mediated gene silencing (Figure 4f). In mammalian cells, microRNAs are processed by Drosha and Dicer to form mature microRNAs, which then recruit RNA-induced silencing complex (RISC) to silence their complementary mRNAs. The activity of microRNAs can be modulated by integrating with metabolite-binding aptamers, which inhibit Drosha processing upon metabolite binding thus reducing gene silencing, as demonstrated for the sensing of theophylline, hypoxanthine, and tetracycline (Beisel et al., 2011).

Although the number of output mechanisms of RNA sensors keeps growing, only a small number of RNA sensors are used in metabolic engineering (Gredell et al., 2012), partially due to the lack of aptamers for target metabolites. Another challenge to engineer RNA-based biosensors is the linkage between an aptamer and an actuation domain, which often requires extensive tuning to correlate metabolite-binding to actuation. To address this problem and make the design of RNA-based metabolite biosensors more extensible, a framework for the modular assembly of aptamers, linkers and gene regulatory components was created that allows simple swapping of aptamer domains between riboswitches (Win and Smolke, 2007). More recently, synthetic biologists have developed computational tools to rationally design riboswitches using existing aptamers that could provide relative predictable control over gene expression, which might have broad 
applications in metabolic engineering (Espah Borujeni et al., 2014).

\subsection{Protein Activity-Based Biosensors}

Protein activity-based biosensors couple a metabolite interaction to the intramolecular modulation of enzymatic or fluorescent activity. Multiple strategies have emerged to accomplish the task of creating novel metabolitebinding domain and actuator domain pairs for new functionality (Yu et al., 2015). Protein activity-based biosensors with fluorescent outputs are highly useful in quantifying metabolic dynamics of engineered microbial strains and have been designed to sense an impressive variety of metabolites (Frommer et al., 2009). For example, Forster Resonance Energy Transfer (FRET) based sensors use metabolite-induced protein conformational changes to alter the efficiency of energy transfer between two fused fluorescent proteins, giving a measurable ratiometric index of a metabolite concentration (Figure 5a). Recently developed FRET proteins include sensors of glutamine (Behjousiar et al., 2012), trehalose-6-phosphate (Peroza et al., 2015), and redox cofactors (Hung et al., 2011). The sophistication of FRET sensors also continues to improve. Of particular note, Ding et al. developed fluorescent protein exchange (FPX) biosensors using dimerization-dependent fluorescence proteins to measure protease activity, second messenger signaling, and intracellular calcium (Ding et al., 2015). These FPX biosensors operate on the principle of the competitive binding of two fluorescent protein monomers (GF and $\mathrm{RF}$ ) to a third, activating monomer (AM). Both $\mathrm{AM}$ and RF are fused to ligand-dependent interacting proteins or peptides so that in the absence of the 
ligand, AM interacts with GF and emits strong green fluorescence. The presence of the target ligand recruits $\mathrm{AM}$ to $\mathrm{RF}$, thus emitting red fluorescence and causing a dramatic fluorescent shift (Figure 5b). Although the ratiometric FRET signals provide higher accuracy than the use of a single fluorophore, both signal strength and ratios are still very sensitive to environmental changes (Constantinou and Polizzi, 2013). Great care must be taken to ensure changes in factors such as $\mathrm{pH}$, salt, and temperature are not misinterpreted as changes in the metabolite of interest (Moussa et al., 2014).

One protein family particularly useful in engineering new protein activitybased sensors is the periplasmic binding proteins (PBPs). A PBP contains a ligand binding domain in its hinge region such that a novel metabolite-binding domain can easily be swapped to induce changes in consequent enzymatic or fluorescent activities (Dwyer and Hellinga, 2004). Alternatively, periplasmic binding proteins with preexisting abilities to bind metabolites of interest can be flanked with FRET components to create a novel biosensor, as demonstrated by the creation of a LivK PBP leucine biosensor (Mohsin et al., 2013).

Significant further potential of protein-activity based sensors lies in actuator functions able to enzymatically regulate engineered metabolic pathways (e.g. allosterically regulated enzymes). Chen et al. elegantly modified homoserine dehydrogenase, an enzyme competing with the L-lysine production pathway for precursors, to act as a biosensor of L-lysine that turns off a competing pathway during L-lysine production (Chen et al., 2015). The inhibition of a competing pathway by a desired product is a prime example of altering 
endogenous biosensing to increase yield. Further, Choi et al. (Choi and Ostermeier, 2015) created a TEM-1 $\beta$-lactamase fusion protein with novel logic gate activity responsive to both redox state and maltose binding. Although this sensor was not applied to bioproduction, nitrocefalin hydrolysis (index of $\beta$-lactamase activity) regulated by these factors established proof of concept for how complex biosensing activities and useful enzymatic outputs can be integrated in one protein.

Challenges in designing protein sensors include maintaining sensor selectivity and output activity in response to metabolite binding. Rational design and computational tools (Grisewood et al., 2013) can provide a starting point for a novel binding or catalytic domain in an existing protein, and directed evolution approaches can further improve sensor selectivity and dynamic range (Ostermeier, 2005). More strategies for designing novel allosterically regulated enzymes were discussed in a recent review (Makhlynets et al., 2015). Clearly, there is great opportunity for incorporating post translational biosensing mechanisms into metabolic pathways for increases in productivity. The fact that post translational regulation is particularly common in the regulation of central metabolic pathways (Pisithkul et al., 2015) hints at the need to target these mechanisms for optimal diversion of resources towards chemical production. We expect that continued research into the rich world of natural allosteric regulation will provide targets for creating protein-based biosensors useful for metabolic engineering. With no doubt, the conceptual knowledge of how these existing systems work will inform the creation of novel sensor-actuator couplings. 


\section{Discussion and Future Directions}

Recent years have seen major innovations in the development of metabolite biosensors with increasing numbers of metabolite targets, mechanisms of action, and applications in metabolic engineering. In addition to specific advantages and disadvantages discussed thus far, several additional considerations can help to broadly compare sensors and determine which may be most appropriate for a given application (Table 1).

One such consideration involves the chemical nature of the metabolitebinding domain. Nucleic acid based sensors (riboswitches, ribozymes) feature limited chemical diversity which might limit their existing and potential metabolite sensing capabilities, although technologies such as RNA SELEX may allow rapid exploration of the functional sequence space (Ellington and Szostak, 1990). Protein-based sensors (TCS, MRTF, FRET, etc) feature much more chemical diversity due to more functional groups in proteins and the vast number of protein folds (Stein and Alexandrov, 2015). These properties ultimately offer a higher potential to protein-based biosensors in their diversity, sensitivity, and specificity in metabolite-binding capabilities. Conversely, classes of metabolite biosensors with actuator domains based on or interacting with nucleic acid chemistry (Riboswitches, ribozymes, MRTF's, TCS-RR) have the distinct advantage of much more predictable outputs. Characteristics can be readily tuned by mutating the sequences, location, and/or copy number of regulatory DNA/RNA sequences to modulate expression levels (Cox et al., 2007). On the other hand, linking metabolite 
binding to novel, desirable changes in protein properties (FRET, enzymatic activities, TCS-HK) is substantially more challenging. Protein folding, metabolite binding-induced conformational changes, and intra- or intermolecular signal transduction are currently harder to predict and engineer than nucleic acid-based chemistry. Successful approaches often require multiple rounds of complementary computational, experimental, and directed evolution approaches (Looger et al., 2003). This may be one reason why metabolite biosensors have not expanded into many other enzymatic outputs that may be useful for dynamic regulation.

A second consideration for biosensors in metabolic engineering applications is the response time from metabolite sensing to actuation. TCS, MRTF, and RNA sensors act at the transcriptional or translational level; therefore readable, selectable, or regulatory phenotype may lag minutes to hours behind actual metabolite changes. Protein activity-based sensors (ex: FRET) respond in the range of milliseconds to seconds. Whether this shorter response time offers substantial advantages in metabolic engineering depends on the nature of application. Rapid responses may have been driven by evolutionary fitness advantages to abrupt, large magnitude changes in nature, or through the ability to better regulate relatively toxic, high-flux metabolic intermediates (Gerosa and Sauer, 2011). On the other hand, in a soundly designed, generally stable bioreactor culture, changes in metabolites themselves are often slow, and drastic committed changes to acute changes in metabolite levels may be undesirable. In such cases, very rapid sensing may not be significantly advantageous. 
Third, it is well documented that synthesis of RNAs and proteins inevitably consumes cellular resources that could be otherwise used for product synthesis and cellular growth. In this regard, RNA-based metabolite biosensors targeting translation (ribozymes and riboswitches) may be superior to those requiring more initial investment in RNA and protein synthesis for responses (MRTF's, TCS, FRET). Indeed, this possibility has been explored by a theoretical work that designed RNA-based genetic control systems to improve the production of engineered metabolic pathways (Stevens and Carothers, 2015). In any case, tuning biosensor expression levels to the minimum necessary to sense metabolites and to exhibit desired actuator activity will minimize the metabolic burden on the cell.

The approaches outlined thus far have already greatly improved the microbial production of many compounds, making the engineering and optimization of metabolic pathways more efficient and microbial production more economically competitive. However, questions still remain in order to further our understanding of metabolite biosensors and to better use them in metabolic engineering. How can the variety of sensed metabolites be efficiently expanded? How rapidly does a sensor have to respond for various metabolic engineering applications and how to tune the response time? How can dynamic pathway regulation be used to improve production of various metabolic pathways? Answering these questions will propel the use of metabolite biosensors to the forefront of metabolic pathway design and optimization. 


\section{Acknowledgements}

This work was supported by the National Science Foundation (MCB1453147, MCB1331194), the Defense Advanced Research Projects Agency (D13AP00038), and the International Center for Advanced Renewable Energy and Sustainability (I-CARES).

\section{References}

Alsaker, K. V., Paredes, C., Papoutsakis, E. T., 2010. Metabolite Stress and Tolerance in the Production of Biofuels and Chemicals: Gene-Expression-Based Systems Analysis of Butanol, Butyrate, and Acetate Stresses in the Anaerobe Clostridium acetobutylicum. Biotechnol Bioeng. 105, 1131-1147.

Babiskin, A. H., Smolke, C. D., 2011. Engineering ligand-responsive RNA controllers in yeast through the assembly of RNase III tuning modules. Nucleic Acids Res. 39, 5299-5311.

Behjousiar, A., Kontoravdi, C., Polizzi, K. M., 2012. In Situ Monitoring of Intracellular Glucose and Glutamine in $\mathrm{CHO}$ Cell Culture. Plos One. 7.

Beisel, C. L., Chen, Y. Y., Culler, S. J., Hoff, K. G., Smolke, C. D., 2011. Design of small moleculeresponsive microRNAs based on structural requirements for Drosha processing. Nucleic Acids Res. 39, 2981-2994.

Binder, S., Schendzielorz, G., Stabler, N., Krumbach, K., Hoffmann, K., Bott, M., Eggeling, L., 2012. A high-throughput approach to identify genomic variants of bacterial metabolite producers at the single-cell level. Genome Biol. 13.

Capra, E. J., Laub, M. T., 2012. Evolution of two-component signal transduction systems. Annu Rev Microbiol. 66, 325-47.

Chang, A. L., Wolf, J. J., Smolke, C. D., 2012. Synthetic RNA switches as a tool for temporal and spatial control over gene expression. Curr Opin Biotech. 23, 679-688.

Chen, Z., Rappert, S., Zeng, A. P., 2015. Rational Design of Allosteric Regulation of Homoserine Dehydrogenase by a Nonnatural Inhibitor L-Lysine. ACS synthetic biology. 4, 126-131.

Choi, J. H., Ostermeier, M., 2015. Rational design of a fusion protein to exhibit disulfide-mediated logic gate behavior. ACS synthetic biology. 4, 400-6.

Chou, H. H., Keasling, J. D., 2013. Programming adaptive control to evolve increased metabolite production. Nat Commun. 4, 2595.

Constantinou, A., Polizzi, K. M., 2013. Opportunities for bioprocess monitoring using FRET biosensors. Biochem Soc T. 41, 1146-1151.

Cox, R. S., Surette, M. G., Elowitz, M. B., 2007. Programming gene expression with combinatorial promoters. Mol Syst Biol. 3.

Dahl, R. H., Zhang, F., Alonso-Gutierrez, J., Baidoo, E., Batth, T. S., Redding-Johanson, A. M., Petzold, C. J., Mukhopadhyay, A., Lee, T. S., Adams, P. D., Keasling, J. D., 2013. Engineering dynamic pathway regulation using stress-response promoters. Nat Biotechnol. 31, 1039-46.

Dietrich, J. A., Shis, D. L., Alikhani, A., Keasling, J. D., 2013. Transcription Factor-Based Screens and Synthetic Selections for Microbial Small-Molecule Biosynthesis. ACS synthetic biology. 2, 4758.

Ding, Y., Li, J., Enterina, J. R., Shen, Y., Zhang, I., Tewson, P. H., Mo, G. C., Zhang, J., Quinn, A. M., Hughes, T. E., Maysinger, D., Alford, S. C., Zhang, Y., Campbell, R. E., 2015. Ratiometric biosensors based on dimerization-dependent fluorescent protein exchange. Nat Methods. 12, 
195-8, 3 p following 198.

Dixon, N., Duncan, J. N., Geerlings, T., Dunstan, M. S., McCarthy, J. E. G., Leys, D., Micklefield, J., 2010. Reengineering orthogonally selective riboswitches. P Natl Acad Sci USA. 107, 2830-2835.

Dwyer, M. A., Hellinga, H. W., 2004. Periplasmic binding proteins: a versatile superfamily for protein engineering. Curr Opin Struc Biol. 14, 495-504.

Ellington, A. D., Szostak, J. W., 1990. Invitro Selection of Rna Molecules That Bind Specific Ligands. Nature. 346, 818-822.

Espah Borujeni, A., Mishler, D., Wang, J., Huso, W., Salis, H., Riboswitch calculator. Vol. 2014, https://salis.psu.edu/software/RiboswitchCalculator_EvaluateMode, 2014, pp. Design of synthetic riboswitches from diverse RNA aptamers.

Fiorentino, G., Ronca, R., Bartolucci, S., 2009. A novel E-coli biosensor for detecting aromatic aldehydes based on a responsive inducible archaeal promoter fused to the green fluorescent protein. Appl Microbiol Biot. 82, 67-77.

Fowler, C. C., Brown, E. D., Li, Y. F., 2010. Using a Riboswitch Sensor to Examine Coenzyme B-12 Metabolism and Transport in E. coli. Chem Biol. 17, 756-765.

Frommer, W. B., Davidson, M. W., Campbell, R. E., 2009. Genetically encoded biosensors based on engineered fluorescent proteins. Chem Soc Rev. 38, 2833-2841.

Ganesh, I., Ravikumar, S., Yoo, I. K., Hong, S. H., 2015. Construction of malate-sensing Escherichia coli by introduction of a novel chimeric two-component system. Bioproc Biosyst Eng. 38, 797-804.

Gerosa, L., Sauer, U., 2011. Regulation and control of metabolic fluxes in microbes. Curr Opin Biotech. 22, 566-575.

Gredell, J. A., Frei, C. S., Cirino, P. C., 2012. Protein and RNA engineering to customize microbial molecular reporting. Biotechnology Journal. 7, 477-499.

Grisewood, M. J., Gifford, N. P., Pantazes, R. J., Li, Y., Cirino, P. C., Janik, M. J., Maranas, C. D., 2013. OptZyme: Computational Enzyme Redesign Using Transition State Analogues. Plos One. 8.

Hsiao, V., de los Santos, E. L. C., Whitaker, W. R., Dueber, J. E., Murray, R. M., 2015. Design and Implementation of a Biomolecular Concentration Tracker. ACS synthetic biology. 4, 150-161.

Hung, Y. P., Albeck, J. G., Tantama, M., Yellen, G., 2011. Imaging Cytosolic NADH-NAD(+) Redox State with a Genetically Encoded Fluorescent Biosensor. Cell Metab. 14, 545-554.

Liu, D., Xiao, Y., Evans, B., Zhang, F., 2013. Negative feedback regulation of fatty acid production based on a malonyl-CoA sensor-actuator. ACS synthetic biology.

Looger, L. L., Dwyer, M. A., Smith, J. J., Hellinga, H. W., 2003. Computational design of receptor and sensor proteins with novel functions. Nature. 423, 185-190.

Makhlynets, O. V., Raymond, E. A., Korendovych, I. V., 2015. Design of allosterically regulated protein catalysts. Biochemistry-Us. 54, 1444-56.

Meinhardt, S., Manley, M. W., Jr., Becker, N. A., Hessman, J. A., Maher, L. J., 3rd, Swint-Kruse, L., 2012. Novel insights from hybrid Lacl/GalR proteins: family-wide functional attributes and biologically significant variation in transcription repression. Nucleic Acids Res. 40, 11139-54.

Michener, J. K., Smolke, C. D., 2012. High-throughput enzyme evolution in Saccharomyces cerevisiae using a synthetic RNA switch. Metab Eng. 14, 306-316.

Michener, J. K., Thodey, K., Liang, J. C., Smolke, C. D., 2012. Applications of genetically-encoded biosensors for the construction and control of biosynthetic pathways. Metab Eng. 14, 212222.

Mohsin, M., Abdin, M. Z., Nischal, L., Kardam, H., Ahmad, A., 2013. Genetically encoded FRET-based nanosensor for in vivo measurement of leucine. Biosens Bioelectron. 50, 72-77.

Moussa, R., Baierl, A., Steffen, V., Kubitzki, T., Wiechert, W., Pohl, M., 2014. An evaluation of genetically encoded FRET-based biosensors for quantitative metabolite analyses in vivo. J Biotechnol. 191, 250-259.

Mukherjee, K., Bhattacharyya, S., Peralta-Yahya, P., 2015. GPCR-Based Chemical Biosensors for Medium-Chain Fatty Acids. ACS synthetic biology.

Muranaka, N., Sharma, V., Nomura, Y., Yokobayashi, Y., 2009. An efficient platform for genetic selection and screening of gene switches in Escherichia coli. Nucleic Acids Res. 37.

Mustafi, N., Grunberger, A., Kohlheyer, D., Bott, M., Frunzke, J., 2012. The development and application of a single-cell biosensor for the detection of L-methionine and branched-chain amino acids. Metab Eng. 14, 449-457.

Ostermeier, M., 2005. Engineering allosteric protein switches by domain insertion. Protein engineering, design \& selection : PEDS. 18, 359-64. 
Palmer, A. E., Qin, Y., Park, J. G., McCombs, J. E., 2011. Design and application of genetically encoded biosensors. Trends Biotechnol. 29, 144-152.

Peroza, E. A., Ewald, J. C., Parakkal, G., Skotheim, J. M., Zamboni, N., 2015. A genetically encoded Forster resonance energy transfer sensor for monitoring in vivo trehalose-6-phosphate dynamics. Anal Biochem. 474, 1-7.

Pisithkul, T., Patel, N. M., Amador-Noguez, D., 2015. Post-translational modifications as key regulators of bacterial metabolic fluxes. Curr Opin Microbiol. 24, 29-37.

Polizzi, K. M., Kontoravdi, C., 2015. Genetically-encoded biosensors for monitoring cellular stress in bioprocessing. Curr Opin Biotech. 31, 50-56.

Raman, S., Rogers, J. K., Taylor, N. D., Church, G. M., 2014. Evolution-guided optimization of biosynthetic pathways. P Natl Acad Sci USA. 111, 17803-17808.

Reed, B., Blazeck, J., Alper, H., 2012. Evolution of an alkane-inducible biosensor for increased responsiveness to short-chain alkanes. J Biotechnol. 158, 75-79.

Rutherford, B. J., Dahl, R. H., Price, R. E., Szmidt, H. L., Benke, P. I., Mukhopadhyay, A., Keasling, J. D., 2010. Functional Genomic Study of Exogenous n-Butanol Stress in Escherichia coli. Appl Environ Microb. 76, 1935-1945.

Salis, H., Tamsir, A., Voigt, C., 2009. Engineering bacterial signals and sensors. Contributions to microbiology. 16, 194-225.

Schallmey, M., Frunzke, J., Eggeling, L., Marienhagen, J., 2014. Looking for the pick of the bunch: highthroughput screening of producing microorganisms with biosensors. Curr Opin Biotech. 26, 148-154.

Shis, D. L., Hussain, F., Meinhardt, S., Swint-Kruse, L., Bennett, M. R., 2014. Modular, multi-input transcriptional logic gating with orthogonal Lacl/GalR family chimeras. ACS synthetic biology. 3, 645-51.

Shong, J., Diaz, M. R. J., Collins, C. H., 2012. Towards synthetic microbial consortia for bioprocessing. Curr Opin Biotech. 23, 798-802.

Stein, V., Alexandrov, K., 2015. Synthetic protein switches: design principles and applications. Trends Biotechnol. 33, 101-110.

Stevens, J. T., Carothers, J. M., 2015. Designing RNA-Based Genetic Control Systems for Efficient Production from Engineered Metabolic Pathways. ACS synthetic biology. 4, 107-115.

Su, L. A., Jia, W. Z., Hou, C. J., Lei, Y., 2011. Microbial biosensors: A review. Biosens Bioelectron. 26, 1788-1799.

Tang, S. Y., Cirino, P. C., 2011. Design and Application of a Mevalonate-Responsive Regulatory Protein. Angew Chem Int Edit. 50, 1084-1086.

Tang, S. Y., Fazelinia, H., Cirino, P. C., 2008. AraC regulatory protein mutants with altered effector specificity. J Am Chem Soc. 130, 5267-5271.

Tang, S. Y., Qian, S., Akinterinwa, O., Frei, C. S., Gredell, J. A., Cirino, P. C., 2013. Screening for Enhanced Triacetic Acid Lactone Production by Recombinant Escherichia coli Expressing a Designed Triacetic Acid Lactone Reporter. J Am Chem Soc. 135, 10099-10103.

Topp, S., Reynoso, C. M. K., Seeliger, J. C., Goldlust, I. S., Desai, S. K., Murat, D., Shen, A., Puri, A. W., Komeili, A., Bertozzi, C. R., Scott, J. R., Gallivan, J. P., 2010. Synthetic Riboswitches That Induce Gene Expression in Diverse Bacterial Species. Appl Environ Microb. 76, 7881-7884.

Trausch, J. J., Ceres, P., Reyes, F. E., Batey, R. T., 2011. The Structure of a Tetrahydrofolate-Sensing Riboswitch Reveals Two Ligand Binding Sites in a Single Aptamer. Structure. 19, 1413-1423.

Van Dorst, B., Mehta, J., Bekaert, K., Rouah-Martin, E., De Coen, W., Dubruel, P., Blust, R., Robbens, J., 2010. Recent advances in recognition elements of food and environmental biosensors: $A$ review. Biosens Bioelectron. 26, 1178-1194.

Wachsmuth, M., Findeiss, S., Weissheimer, N., Stadler, P. F., Morl, M., 2013. De novo design of a synthetic riboswitch that regulates transcription termination. Nucleic Acids Res. 41, 25412551.

Weigand, J. E., Suess, B., 2007. Tetracycline aptamer-controlled regulation of pre-mRNA splicing in yeast. Nucleic Acids Res. 35, 4179-4185.

Wen, Z. Q., Wu, M. B., Lin, Y. J., Yang, L. R., Lin, J. P., Cen, P. L., 2014. Artificial symbiosis for acetonebutanol-ethanol $(\mathrm{ABE})$ fermentation from alkali extracted deshelled corn cobs by co-culture of Clostridium beijerinckii and Clostridium cellulovorans. Microb Cell Fact. 13.

Whitaker, W. R., Davis, S. A., Arkin, A. P., Dueber, J. E., 2012. Engineering robust control of twocomponent system phosphotransfer using modular scaffolds. P Natl Acad Sci USA. 109, 
18090-18095.

Win, M. N., Smolke, C. D., 2007. A modular and extensible RNA-based gene-regulatory platform for engineering cellular function. P Natl Acad Sci USA. 104, 14283-14288.

Xu, P., Wang, W. Y., Li, L. Y., Bhan, N., Zhang, F. M., Koffas, M. A. G., 2014. Design and Kinetic Analysis of a Hybrid Promoter-Regulator System for Malonyl-CoA Sensing in Escherichia coli. ACS chemical biology. 9, 451-458.

Yu, K., Liu, C. C., Kim, B. G., Lee, D. Y., 2015. Synthetic fusion protein design and applications. Biotechnol Adv. 33, 155-164.

Zhang, F. Z., Carothers, J. M., Keasling, J. D., 2012. Design of a dynamic sensor-regulator system for production of chemicals and fuels derived from fatty acids. Nat Biotechnol. 30, 354-U166.

Zhang, F. Z., Keasling, J., 2011. Biosensors and their applications in microbial metabolic engineering. Trends Microbiol. 19, 323-329.

Zhou, K., Qiao, K., Edgar, S., Stephanopoulos, G., 2015. Distributing a metabolic pathway among a microbial consortium enhances production of natural products. Nat Biotechnol. 33, 377-83.

Zhou, L. B., Zeng, A. P., 2015. Exploring Lysine Riboswitch for Metabolic Flux Control and Improvement of I-Lysine Synthesis in Corynebacterium glutamicum. ACS synthetic biology. 
Figure 1. Applications of metabolite biosensors in metabolic engineering. a) Biosensors can be linked to a colorimetric output to report the concentration of metabolites, providing a straightforward way to screen for high-producing strains. b) Biosensors can be used to control an output associated with a fitness advantage under selective conditions. This allows direct enrichment and selection of high-producers. c) Biosensors can be used to control the activity of a metabolic pathway, which allows dynamic optimization of the pathway activity according to the level of the sensed metabolite.

a.

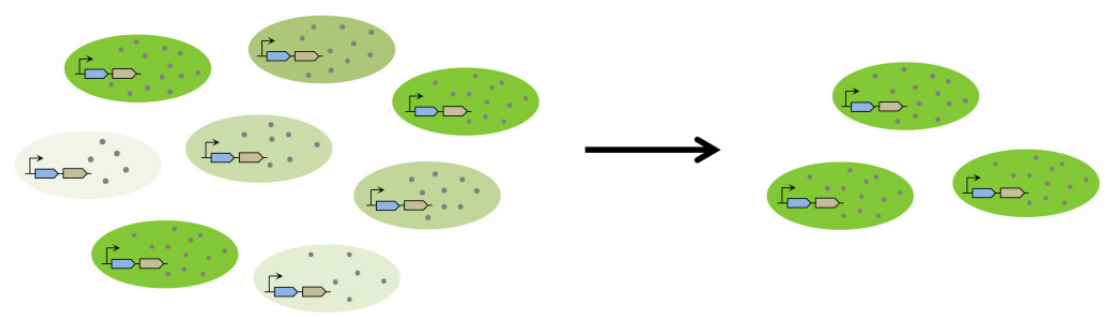

b.

Selection

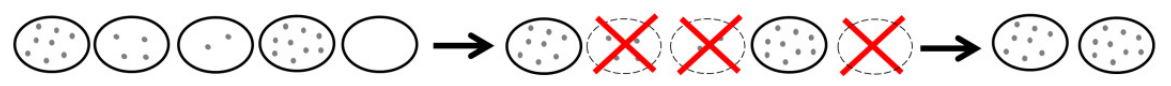

c.

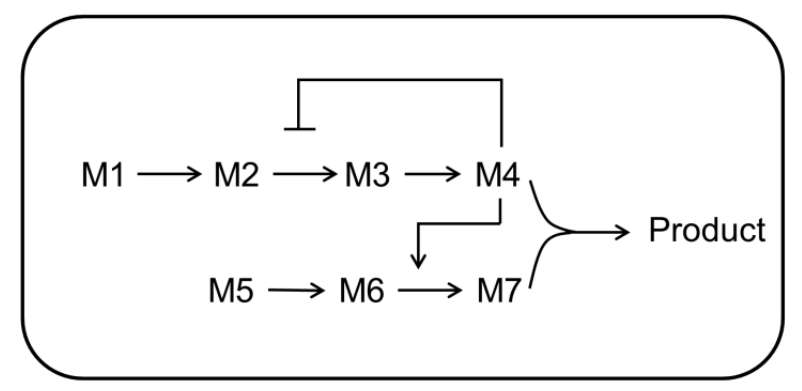


Figure 2. Metabolite-responsive transcription factor (MRTF)-based metabolite biosensors and examples of their applications in metabolic engineering. a) Metabolite binding to an MRTF represses transcription. b) Metabolite binding to an MRTF enhances transcription. c) A dynamic pathway regulation system for fatty acid ethyl ester (FAEE) production. Acyl-CoA biosensors were used to activate synthesis of FAEE and its precursors, ethanol and fatty acyl-CoA. This synthetic regulatory system dynamically turns on downstream enzyme expression only when the upstream pathway accumulates enough key intermediates, in this case acyl-CoA.

a.

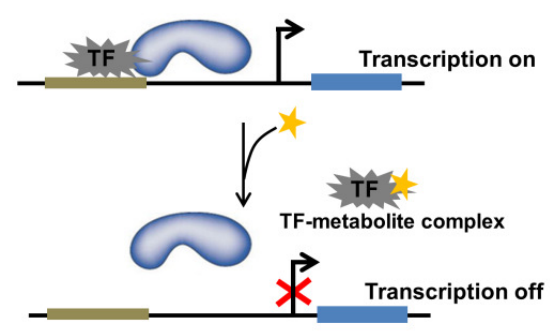

b.

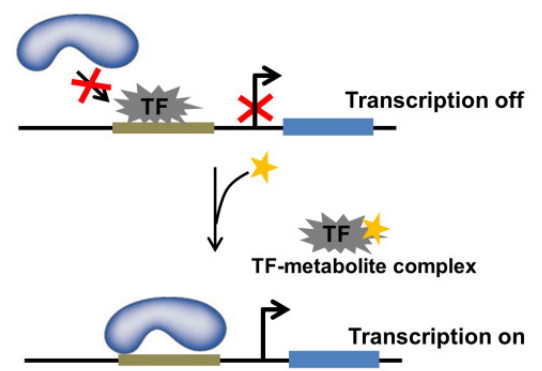

c.

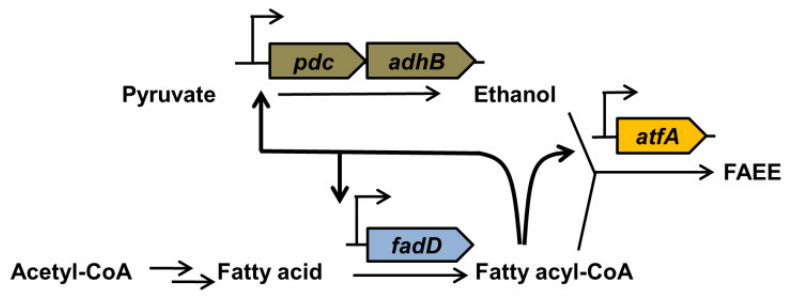


Figure 3. Biosensors based on two-component systems and stress response. a) A two-component system consists of a histidine kinase (HK) domain and a response regulator (RR) domain. Detection of an extracellular metabolite autophosphorylates the HK, which regulates the phosphorylation of the cognate RR. The cognate RR then controls the expression of a gene. b) Promoters that are modulated by metabolite-induced cellular stress can be identified from transcriptomic analysis. Promoters that are differentially expressed in the presence of the metabolite can be cloned to control the expression of a reporter. c) An example of using stress-response-based biosensor for metabolic engineering. Biosensors for FPP were identified from RNA microarray and used to construct a dynamic regulation circuit for amorphadiene production. Promoters that are repressed by FPP were used to down-regulate FPP synthesis, and promoters that are activated by FPP were used to up-regulate a downstream gene ads, whose enzyme product converts FPP to amorphadiene.

a.

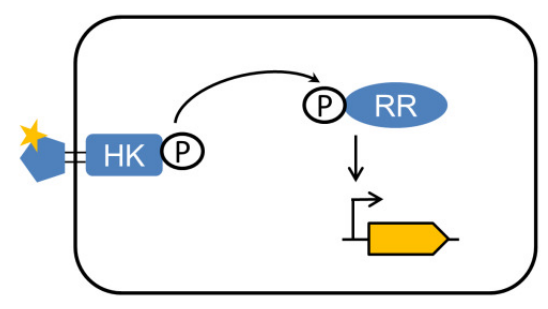

b.

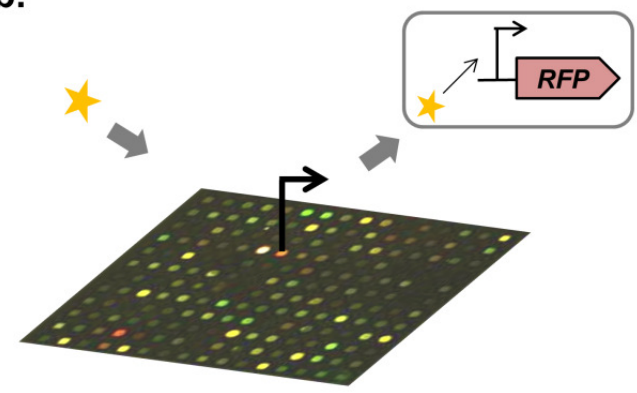

c.

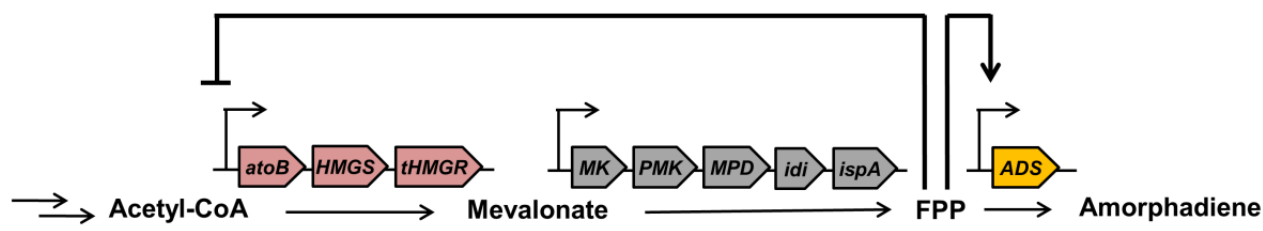


Figure 4. RNA-based metabolite biosensors. The gene expression can be turned up or turned down upon sensing the metabolite, and in each case one example is shown. a) A metabolite biosensor based on metabolite-controlled transcription termination. The presence of the metabolite disrupts the terminator structure, leading to gene activation. b) A metabolite biosensor based on modulating translation initiation. Binding of the metabolite sequesters the RBS from the ribosome, leading to decreased gene expression. c) An aptazyme-based metabolite biosensor that modulates mRNA cleavage activity. Binding of the metabolite stabilizes an inactive ribozyme conformation, which inhibits the ribozyme cleavage. The arrow indicates the ribozyme cleavage site. d) An Rnt1p switch-based metabolite biosensor which inhibits the cleavage activity of an RNase III enzyme upon metabolite binding. e) A metabolite biosensor based on RNA splicing. Binding of the metabolite inhibits the splicing, leading to increased gene expression. f) A metabolite biosensor based on microRNAs. Integration of aptamers with microRNAs inhibits the maturation of microRNAs upon metabolite binding, which reduces gene silencing and leads to higher gene expression.

a.

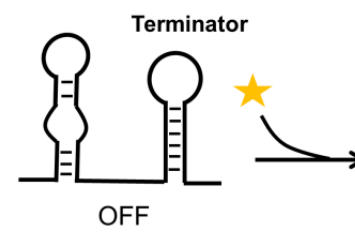

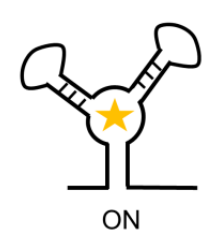

b.

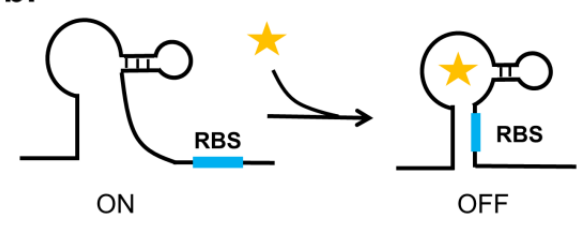

d.

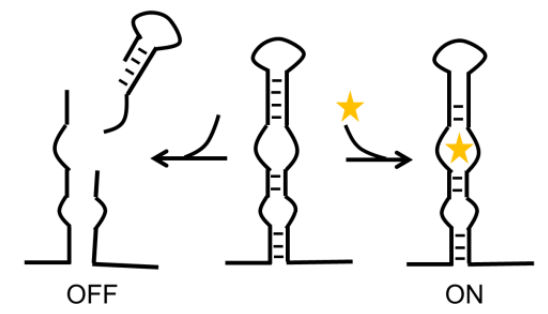

e.

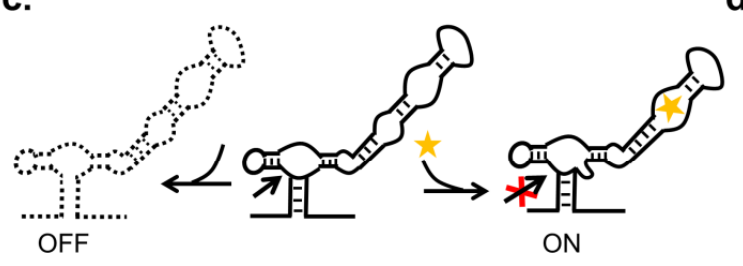

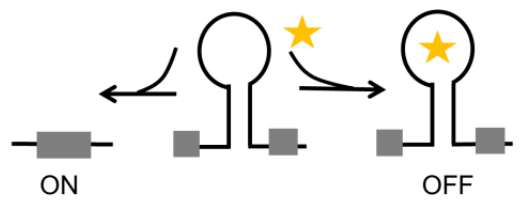

f.

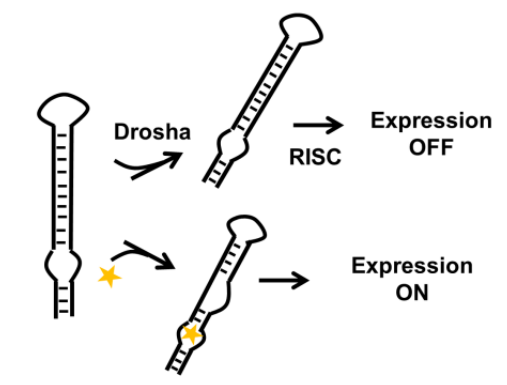


Figure 5. Protein-activity based metabolite biosensors. a) A FRET biosensor that reports the binding of a metabolite as a change in the FRET signals between a fluorophore pair. b) A fluorescence protein exchange (FPX) biosensor. GF and RF represent green and red fluorescence protein monomers, respectively. AM represents an activating monomer that increases the fluorescence of GF and RF when they bind to form heterodimers. The binding of the ligand alters the interaction of AM to GF and RF by triggering a ligand-dependent protein interaction, leading to a change in the fluorescence ratio. c) A lysine biosensor was engineered to allosterically inhibit the enzyme activity of homoserine dehydrogenase, which is naturally inhibited by threonine. This engineered sensor turned down the conversion of aspartyl- $\beta$ semialdehyde to homoserine, thus decreasing the byproduct formation in lysine synthesis.

a.

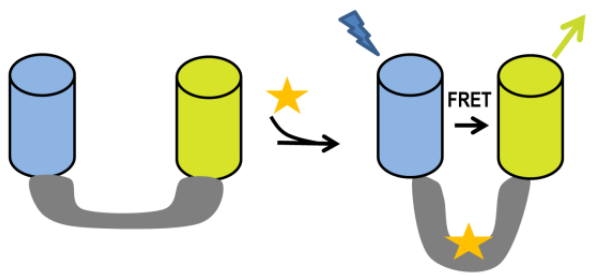

b.

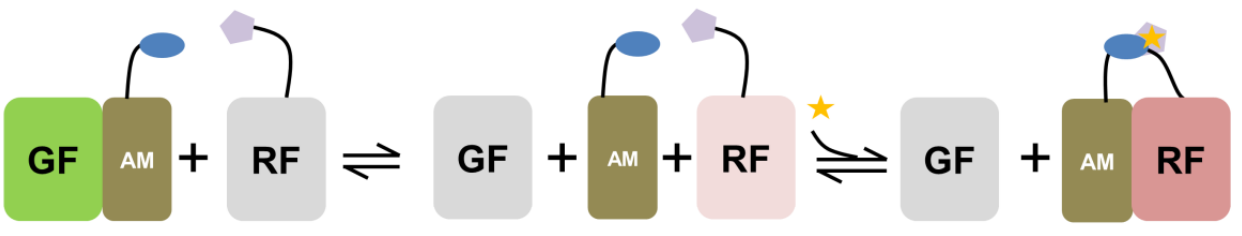

c.

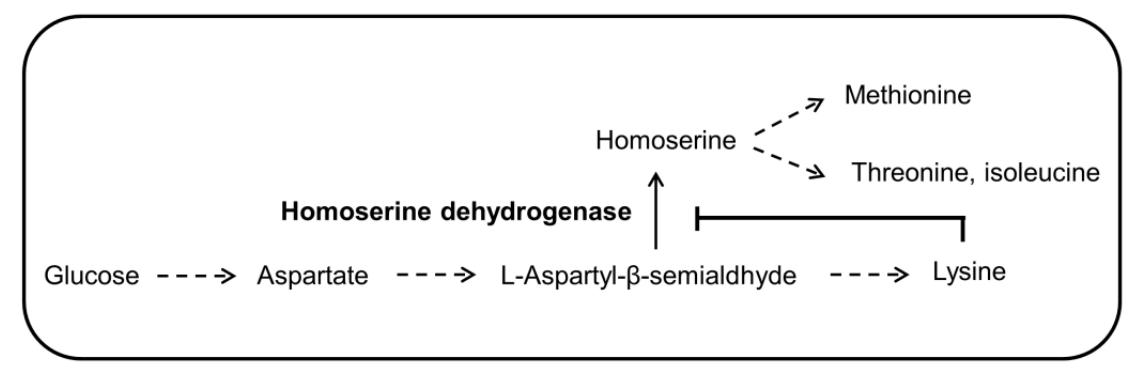


Table 1. Comparison of advantages and disadvantages of metabolite biosensors in each of the five categories in terms of ease of tuning, specificity, response time and metabolite molecular diversity.

\begin{tabular}{|c|c|c|c|c|c|}
\hline $\begin{array}{c}\text { Biosensor } \\
\text { Type }\end{array}$ & $\begin{array}{l}\text { Ease of } \\
\text { tuning }\end{array}$ & Specificity & $\begin{array}{c}\text { Response } \\
\text { Time }\end{array}$ & $\begin{array}{c}\text { Metabolite } \\
\text { molecular } \\
\text { diversity }\end{array}$ & Example Refs \\
\hline MRTFs & Easy & $\begin{array}{l}\text { Medium to } \\
\text { High }\end{array}$ & Slow & Broad & $\begin{array}{c}\text { (Reed et al., 2012; Tang } \\
\text { and Cirino, 2011; Zhang } \\
\text { et al., 2012) }\end{array}$ \\
\hline $\begin{array}{l}\text { Two- } \\
\text { component } \\
\text { systems }\end{array}$ & Medium & Medium & Slow & Medium & (Ganesh et al., 2015) \\
\hline $\begin{array}{c}\text { Stress } \\
\text { response }\end{array}$ & Difficult & Low & Slow & Broad & (Dahl et al., 2013) \\
\hline RNAs & Easy & High & $\begin{array}{l}\text { Slow to } \\
\text { medium }\end{array}$ & Narrow & $\begin{array}{l}\text { (Michener and Smolke, } \\
\text { 2012; Topp et al., 2010; } \\
\text { Wachsmuth et al., 2013) }\end{array}$ \\
\hline $\begin{array}{l}\text { Protein } \\
\text { activity }\end{array}$ & Difficult & High & Fast & Broad & $\begin{array}{c}\text { (Behjousiar et al., 2012; } \\
\text { Chen et al., 2015; Ding } \\
\text { et al., 2015) }\end{array}$ \\
\hline
\end{tabular}

